\title{
ALA-mediated Photodynamic Therapy Decreases the Ability of Cellular Invasion in CL1-5 Human Lung Adenocarcinoma Cells
}

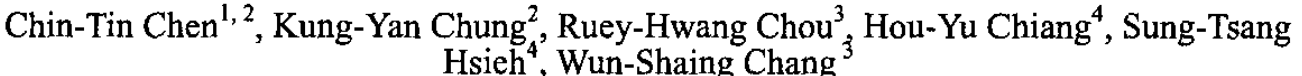 \\ ${ }^{1}$ Center for Optoelectronic Biomedicine, ${ }^{2}$ Graduate Institute of Oral Biology and ${ }^{4}$ Department \\ of Anatomy and Cell Biology, College of Medicine, National Taiwan University; Taipei 100, \\ ${ }^{3}$ National Health Research Institutes, Taipei 115, Taiwan \\ Tel \& Fax: 2-23949140 E-mail: ctchen@ha.mc.ntu.edu.tw
}

\begin{abstract}
In this study, we investigate the ALA-PDT induced biological responses in CL1-5 human lung adenocarcinoma cells. Under ALA-PDT, we found that mitochondrial photodamage results in phenotypic changes and reduced invasive behavior in CL1-5 cells.
\end{abstract}

\section{INTRODUCTION}

Photodynamic therapy (PDT) has been developed as a modality for cancer treatment, which is based on the administration of photosensitizers to induce cytotoxicity after light irradiation (1). It has been shown that singlet oxygen and other reactive oxygen species are responsible for the PDT-induced cell killing that leads to tumor ablation $(1,2)$. As the primary oxidant associated with the PDT reaction is singlet oxygen, photosensitization has been found to oxidize cellular macromolecules including lipids, proteins, and nucleic acids that result in the damage of subcellular structures (3). The sensitivity of cells to PDT varies between cell types, even between very close related cell lines (4-5). In addition to its cytotoxic effect, PDT can act as a form of exogenous stress signal to regulate cellular responses involving the modifications in the signal transduction events leading to gene transcription (6-9).

Mitochondria have traditionally been regarded as the power house in eukaryotic cells. In addition to ATP production, mitochondria also participate in many essential cellular functions, such as ion homeostasis, cell motility, cell proliferation and apoptosis (10-13). Although relatively small in number, mitochondrial-encoded proteins play crucial roles in either the catalytic function or assembly of mitochondrial electron transport chain complexes. Changes in the mitochondrial genome have been implicated in carcinogenesis and other diseases $(12,13)$. In addition, it has been shown that mitochondrial DNA (mitDNA) may play an important role in cellular sensitivity to cancer therapeutic agents (13). These studies indicate the importance of the mitochondria and its genome in cancer and cancer therapy.Mitochondria have been implicated as a primary target of PDT (14). However, the importance of mitDNA in PDT is not clear. Here we present that mitochondrial dysfunctions induced by ALA-PDT result in morphological changing, cytoskeleton reorganization, and increased adherence to substratum. Furthermore, ALA-PDT selected CL1-5 variants display phenotypic changes and decreased invasive behavior.

\section{RESULTS}

Previously, we have found that mitochondria photodamages induced by ALA-PDT could aiter the overall topology of cells and result in increased cellular adhesion to substratum. As alternation of cellular adherence affects the migration of cells, we further assessed the photodynamic effect on the cellular invasion of CL1-5 lung adenocarcinoma cell line, which has been shown having strong invasive ability (15). Under ALA-PDT, we observed the mitochondrial photodamages as revealed by breakdown of mitochondrial membrane potential (Figure 1A) and electron microscopic analysis in CL1-5 cells (data not shown here). As shown in Fig.1B, PDT-induced cytotoxicity did not increase in a fluence-dependent manner, which suggests CL1-5 cells were resistant to ALA photodynamic treatment. The survival rate of CL1-5 cells was also not significantly decreased as the ALA concentration increased (data not shown here). Although CL1-5 cells show resistance to ALA-PDT, significant numbers of cells show round-up (Fig 1C) and increased adherence to substratum. As shown in the right panel of Figure 2, CL1-5 cells without ALA-PDT could be completely removed by $0.05 \%$ of trypsin-EDTA after 3 minutes incubation. However, under the same trypsinization condition, over $50 \%$ of cells still remains in the petri dish 5 minutes post ALA-PDT $\left(6 \mathrm{~J} / \mathrm{cm}^{2}\right)$. Besides, increasing the light dose is associated with an increased resistance to trypsinization. As shown in the left panel of Figure 2, these cellular round up accompanies $\mathrm{F}$-actin reorganization and $\mathrm{N}$-acetyl-cysteine (NAC), free radical scanvanger, inhibits PDT-induced resistance to trypsinization and F-actin reorganization. To address whether this increased adherence affects the migration or invasion of cancer cells, we have CL1-5 cells treated with ALA-PDT. After consecutive 5 times of 


\section{TH2A-SS-8}

ALA-PDT, selected CL1-5 variants were established. Using matrigel migration assay, we found that the ability of invasion in ALA-PDT selected CL1-5 variants were significantly decreased up to $60 \%$ as shown in Figure 3 . In addition, more compact colony was found in PDT-treated cells. Finally, ALA-PDT invariably causes altered cell morphology and mitochondria to redistribute or aggregate along one-side of the cell as shown in Figure 4.These results indicate that the PDT-induced mitochondrial photodamages induce phenotypic changes and invasive behavior in CL1-5 cells.

\section{FIGURE}
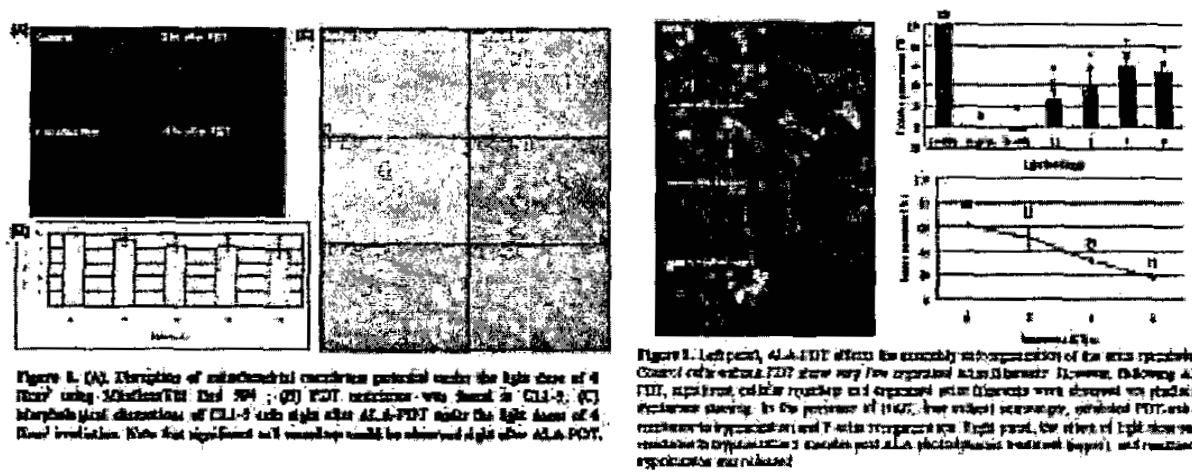

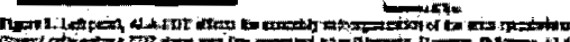

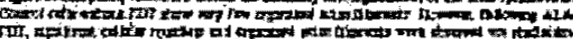

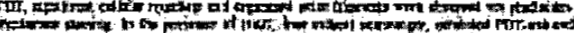

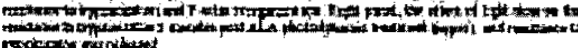
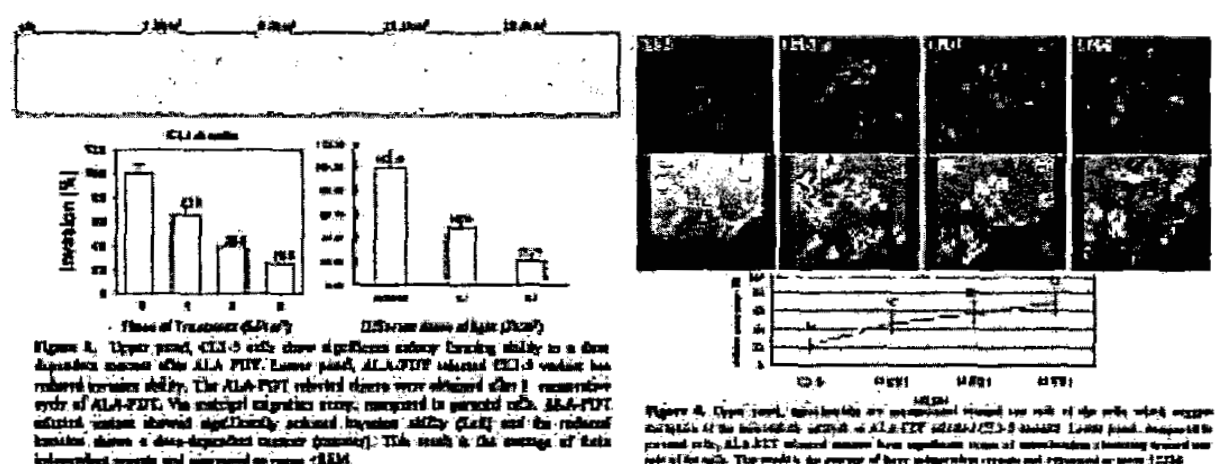

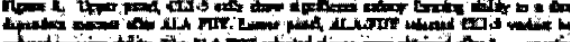

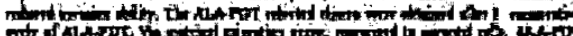
20.

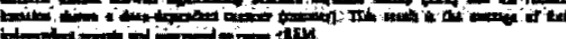

\section{References}

1. Dolmans DEJGJ, Fukumura D, Jain RK. (2003) Nature Reviews Cancer 3:380-387.

2. Schuitmaker JJ, P Baas, HLL van Leengoed et al., (1996) J. Photochem. Photobiol. B:Biology 34: 3-12.

3. Boyle RW, Dolphin D. (1996) Photochem Photobiol, 64:469-485.

4. Agarwal ML, H Larkin, SIA Zaidi et al., (1993) Cancer Res. 53: 5897-5902.

5. Tsuimin Tsai, Ruey-Long Hong, , Pei-Jen Lou, I-Fang Ling and Chin-Tin Chen (2003). Lasers in Surgery and Medicine.34: 62-72.

6. Luna MC, S Wong, and Gomer CJ (1994) Cancer Res. 54:1374-1380.

7. Gomer CJ, A Ferrario, N Rucker et al., (1991) Cancer Res. 51: 6574-6579.

8. Gomer CJ, SW Ryter, A Ferrario, N Rucker et al., (1996) Cancer Res. 56: 2355-2360.

9. Henderson BW and JM Donovan (1989) Cancer Res. 49: 6896-6900.

10. Singh KK. (1998) Mitochondrial DNA mutations in aging, disease and Cancer. Springer: New York, NY, USA

11. Kiberstis PA (1999) Science 283, 1475.

12. Eng C, Kiuru M, Fernandez MJ and Aaltonen LA (2003) Nature Rev Cancer 3:193-202.

13. Singh KV, Russell J, Sigala B et al., (1999) Oncogene 18:6641-6646.

14. Morgan J and Oseroff AR. (2001) Adv. Drug Delivery Reviews. 49:71-86.

15. Chu Y-W, Yang P-C, Yang S-C et al., (1997) Am. J. Respir. Cell Mol. Biol. 17: 353-360. 